\title{
Design of Optical System for Electrostatic Field Distribution Measurement Using Micromirror Array
}

\author{
Toshihide Kuriyama*, Toshikazu Aoi, Hiroshi Maeda ${ }^{1}$, \\ Takaki Itoh $^{1}$, Yoshifumi Ueno ${ }^{1}$, Toshiyuki Nakaie ${ }^{2}$, \\ Nobutika Matsui ${ }^{2}$ and Hiroyuki Okumura ${ }^{2}$ \\ Faculty of Biology-Oriented Science and Technology, Kinki University \\ 930 Nishimitani, Kinokawa-shi, Wakayama 649-6493, Japan \\ 'Industrial Technology Center of Wakayama Prefecture \\ 60 Ogura, Wakayama-shi, Wakayama 649-6261, Japan \\ ${ }^{2}$ Hanwa Electronic Ind. Co., Ltd. \\ 689-3 Ogaito, Wakayama-shi, Wakayama 649-6272, Japan
}

(Received May 2, 2011; accepted June 20, 2011)

Key words: micromirror, electrostatic field distribution, optical system

An optical system for electrostatic field distribution measurement using a micromirror array fabricated by a micro-electromechanical systems (MEMS) process has been designed. Each micromirror is suspended by two thin torsion bars, which is made by a silicon-on-insulator (SOI) wafer fabrication process. The deflection of each mirror by an electrostatic field is measured optically using a two-dimensional optical scanner and a position-sensitive detector (PSD). Another optical system for increasing the sensitivity has also been proposed, which will widen the voltage measurement range. The electrostatic field distribution measurement system was applied to a human body model, and the removal effect of electricity was tested using removing tools.

\section{Introduction}

The malfunction of electronic devices affects human safety considerably. Electronic malfunctions are often caused by electrostatic discharge (ESD), which damages electronic devices such as large integrated circuits (LSIs) and liquid crystal displays (LCDs).

To prevent electronic devices from being destroyed by ESD, the electrostatic distribution measurement of its environment is a useful method. An electric field mill is often used to measure the strength of a static electric field. ${ }^{(1,2)}$ An electrostatic voltmeter based on the electric field mill can be moved along the surface of a charged

"Corresponding author: e-mail: kuriyama@waka.kindai.ac.jp 
body or machine to measure the electrostatic distribution. The moving method of the electrostatic voltmeter, however, takes time and the electrostatic distribution changes during the measurement.

The electrostatic field mills based on micro-electromechanical system (MEMS) technology were developed and could be miniaturized. ${ }^{(3-6)}$ Instead of moving the electrostatic voltmeter, it is possible to integrate the MEMS-based electrostatic voltmeters to measure the electrostatic distribution. The fabrication process and electrical detection circuit, however, will be complicated and expensive.

An electrostatic distribution measurement system, which can measure in a short time (about $1 \mathrm{~s}$ ), has been achieved using a micromirror array, a two-dimensional optical scanner, and a position-sensitive detector (PSD). The micromirror was fabricated by a MEMS process. The measurement system was applied to a human electrical field caused by frictional electricity.

\section{Electrostatic Distribution Measurement System}

\subsection{Principle of electrostatic field measurement}

Coulomb discovered a rule named after him using a torsion balance, that is, the magnitude of the electrostatic force is proportional to the scalar multiplication of the magnitudes of charges and inversely proportional to the square of the distance between them.

$$
F=\mathrm{k}_{\mathrm{e}} \frac{q_{1} q_{2}}{r^{2}}
$$

$F$ is force, $\mathrm{k}_{\mathrm{e}}$ is a proportionality constant (Coulomb constant), $q_{1}$ and $q_{2}$ are point charges, and $r$ is the separation distance between the charges.

The authors compared two micromirror structures to detect the electrostatic force optically, one was a cantilever type and the other was a torsion bar type shown in Fig. 1. The cantilever-type micromirror (a) is supported by cantilevers and has a higher sensitivity by lengthening and making thin the cantilever. ${ }^{(7,8)}$ The sensitivity, however, becomes higher with external mechanical vibration because of its asymmetric structure. On the other hand, the torsion-bar-type micromirror has a symmetric structure and has less sensitivity to external mechanical vibration than the cantilever-type micromirror. The authors adopted the torsion-bar-type micromirror (torsion mirror) and measured the electrostatic force by applying the electrostatic force to half of the micromirror and shielding the other half with a metal plate. The first torsion bar was made of a stainless steel thin wire and a silicon micromirror chip was bonded to the wire to check the detection principle. The torsion bar stiffness $K$ of the stainless steel wire is given from a balance of shearing stress and torsional moment

$$
K=\pi G x^{4} / 32 L
$$

where $G$ is the modulus of rigidity, $x$ is the torsion bar diameter, and $L$ is the torsion bar length. ${ }^{(9)}$ By making thin and lengthening the torsion bar, the sensitivity to electrostatic 


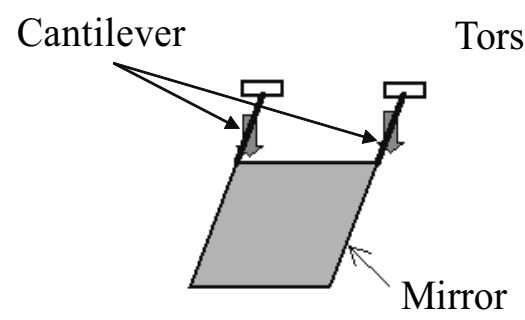

(a)

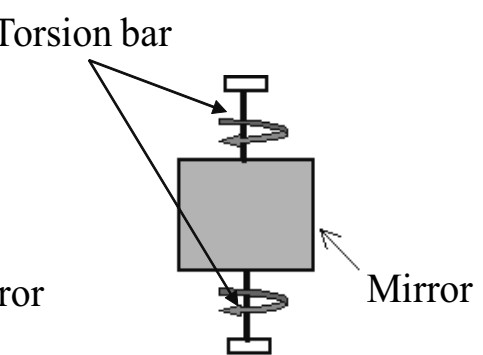

(b)

Fig. 1. Structures for suspending micromirror.

force can be improved. As the first torsion mirror, the stainless steel thin wire was used as a torsion bar and a silicon micromirror chip was bonded to the wire to check the detection principle. The measurement results showed that the deflection of the micromirror is proportional to the square of the applied field; the magnitude, however, did not coincide with the calculated value. The adhesive material used for bonding was thought to make the torsion bar stiffness smaller. To prevent the adhesion problem, the whole torsion mirror was then made of silicon-on-insulator (SOI) wafer by the MEMS process.

\subsection{Micromirror and optical measurement system}

Micromirrors that are suspended by thin wires were fabricated by the MEMS process using an SOI wafer. One half of each micromirror is exposed to an electrostatic field, and the other half is shielded by a metal plate. The micromirrors and metal plate are grounded. Measuring the deflection angle of each micromirror using an optical measurement system enables the electrostatic distribution of charged substances, such as in an LSI fabrication machine and human body. Figure 2 shows the structure and photograph of a torsion mirror where a micromirror is suspended by two torsion bars. The size of the torsion bar is $20 \mu \mathrm{m}$ thick, $20 \mu \mathrm{m}$ wide, and $2 \mathrm{~mm}$ long. The size of the micromirror is $20 \mu \mathrm{m}$ thick, $5 \mathrm{~mm}$ wide, and $5 \mathrm{~mm}$ long.

By applying an electrostatic field to half of the micromirror, the torsion mirror is twisted around the torsion bar. The optical measurement system shown in Fig. 3 was used to measure the deflection angle of the plural micromirrors, i.e., electrostatic distribution. In Fig. 3, the laser beam emitted by a semiconductor laser (wavelength of $650 \mathrm{~nm}$ ) is two-dimensionally scanned using an optical scanner and irradiates perpendicularly each micromirror back surface after passing through the condenser lens. The reflected laser beam at the back surface of the micromirrors passes again through the condenser lens and is reflected by the beam splitter. A PSD detects the position of the reflected laser beam at the PSD surface.

In the optical system, the distance between the convex lens and the two-dimensional scanner is set as the focal distance of the convex lens as shown in Fig. 4. The laser beam is focused on the surface of the two-dimensional scanner; then, it becomes a parallel beam after passing the convex lens and irradiates the micromirrors, where each angle of 

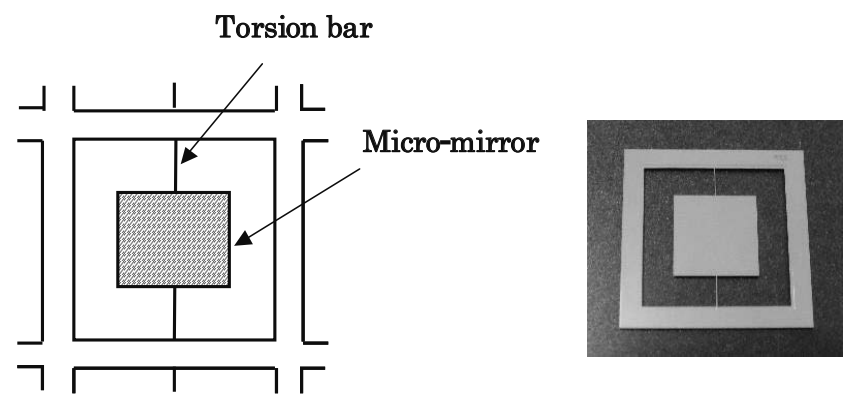

Fig. 2. Structure and photograph of torsion mirror.

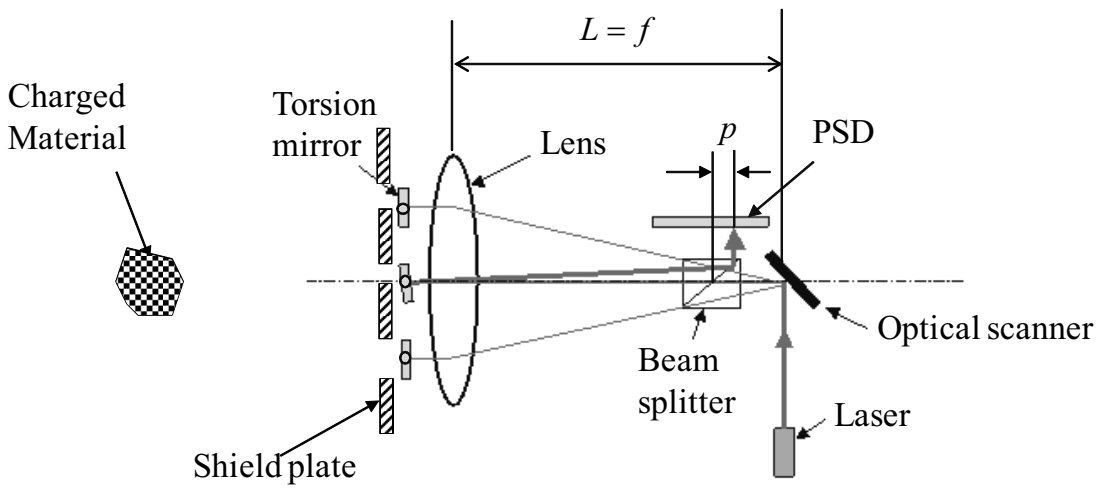

Fig. 3. Optical setup for electrostatic field distribution.

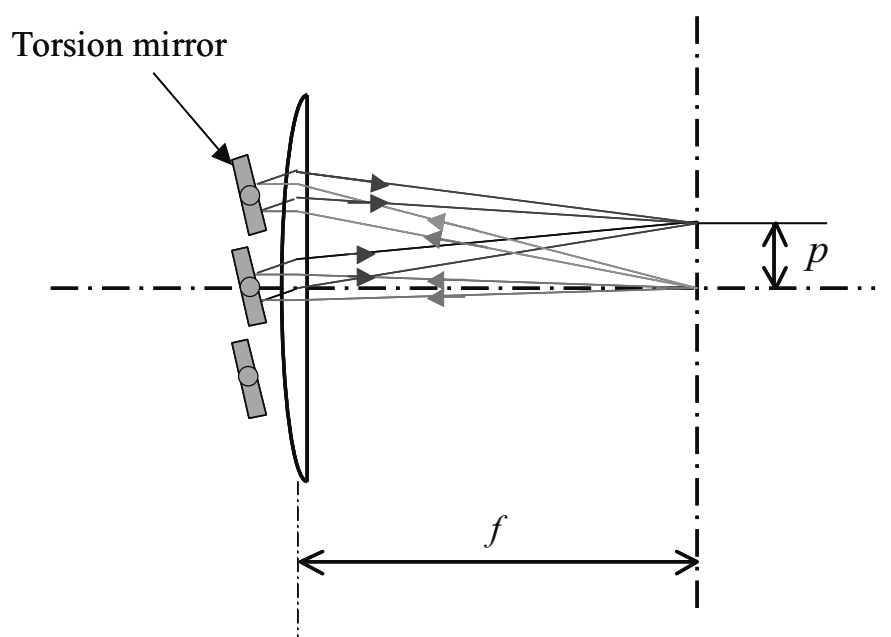

Fig. 4. Principle of torsion mirror deflection angle measurement. 
incidence is perpendicular to the micromirror when the electrostatic field does not exist. When the torsion mirror has a deflection angle $\varphi$ by electrostatic force, the reflection angle $\theta$ of the incident beam is given by

$$
\theta=2 \varphi .
$$

The reflected beam passes through the convex lens again focused on the focal plane at the position $p$ given by

$$
p=f \tan \theta
$$

The relationship given by eq. (4) is applicable to all the laser beams radiated on the micromirrors, and the deflection angle of each micromirror can be measured from the position of the scanned laser beam on the PSD surface.

Figure 5 shows the electrostatic field distribution measurement system. The torsion mirror array that was fabricated using a 4-inch SOI wafer is mounted on a holder and covered by a copper plate that has holes in front of each torsion mirror. A large condenser lens $(150 \mathrm{~mm}$ diameter, $f=300 \mathrm{~mm})$ is set between the torsion mirror array and the two-dimensional optical scanner (Cambridge Technology, Galvano-Scanner Unit

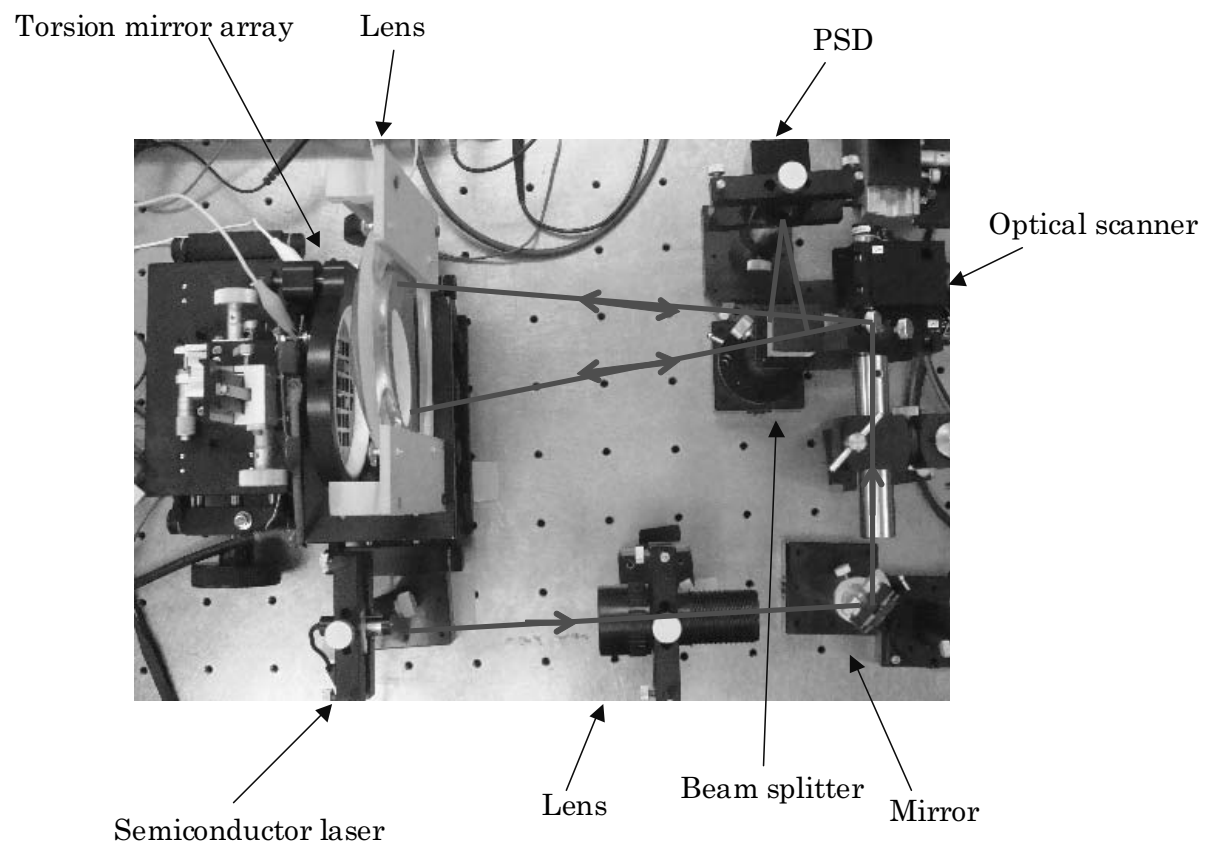

Fig. 5. Electrostatic field distribution measurement system. 
$6215 \mathrm{H})$. A laser beam $(\lambda=650 \mathrm{~nm}$, output power $1 \mathrm{~mW})$ is focused on the horizontal mirror and scanned two-dimensionally, then irradiated on each micromirror through a beam splitter and the large condenser lens. The reflected laser beam is reflected by the beam splitter and is focused on the PSD (Hamamatsu Photonics, C10443-02) surface to measure the spot position $p$.

To check the applicability of the relation given by eq. (4) to each scanned laser beam, the mirror deflection angle and displacement position at the PSD were measured at three different mirror positions (center of convex lens, $2 \mathrm{~cm}$ and $4 \mathrm{~cm}$ peripherally apart from the center). As shown in Fig. 6, three lines have almost the same shape.

Another optical measurement system to improve the sensitivity has also been proposed and its effectiveness was confirmed. The Coulomb force is proportional to the square of the electrostatic voltage of the charged material and very small at a low voltage range as shown in $\S 2.4$. Figure 7 shows the optical system for low electrostatic voltage measurement. An objective lens is placed at a position where the light spot image on the PSD surface is enlarged on another PSD surface. The focal distance of the objective lens $f_{1}$ is chosen to satisfy the relation,

$$
1 / f_{1}=1 / a+1 / b
$$

where $a$ is the distance between the first light spot image and the objective lens and $b$ is the distance between the objective lens and the second light spot image on the second PSD.

The positions of the reflected light spot on the first PSD and second PSD were measured by rotating a mirror at the torsion mirror position. Figure 8 shows that the sensitivity was increased 10 times as high as the sensitivity of the optical system without an objective lens.

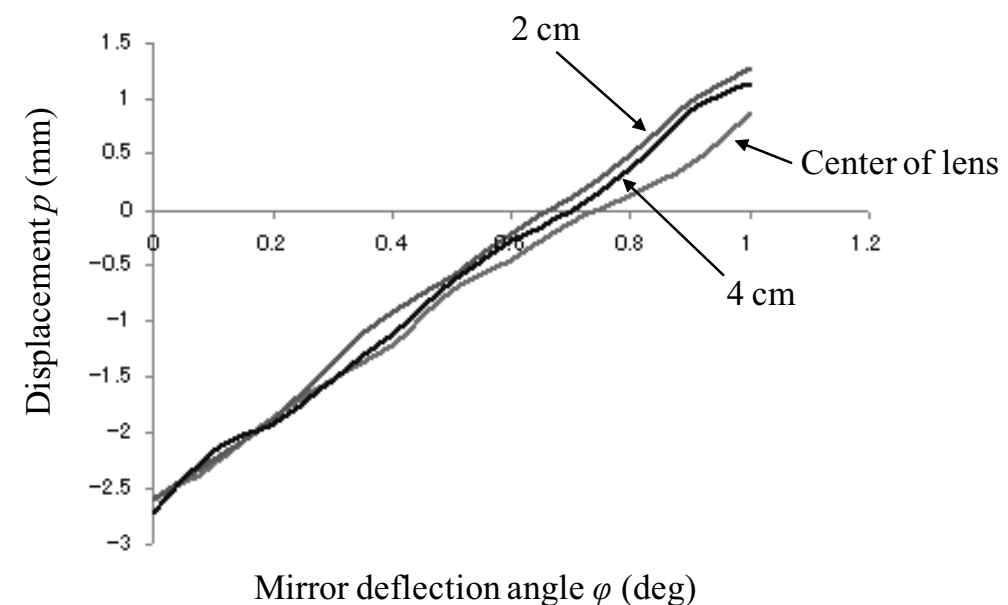

Fig. 6. Relationship between mirror deflection angle and displacement at PSD surface. 


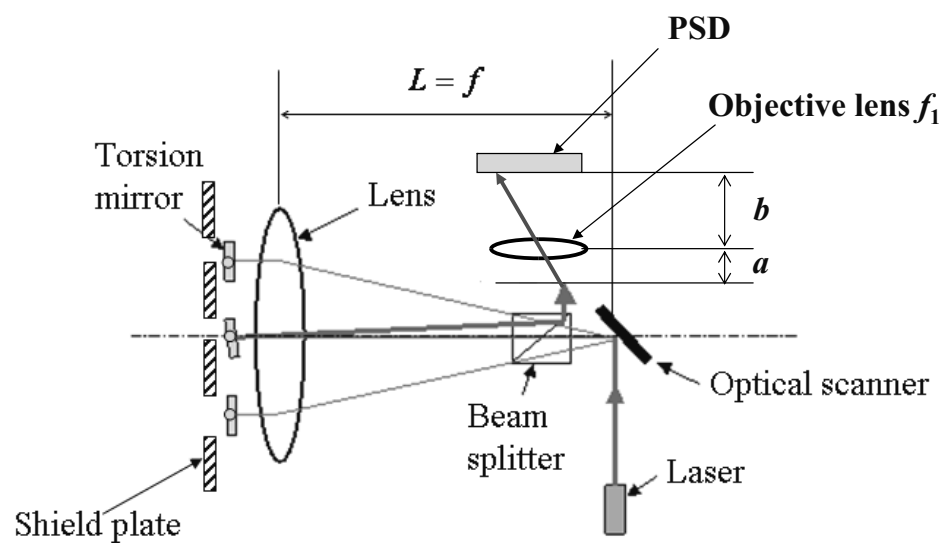

Fig. 7. Principle of optical system for low-voltage measurement.

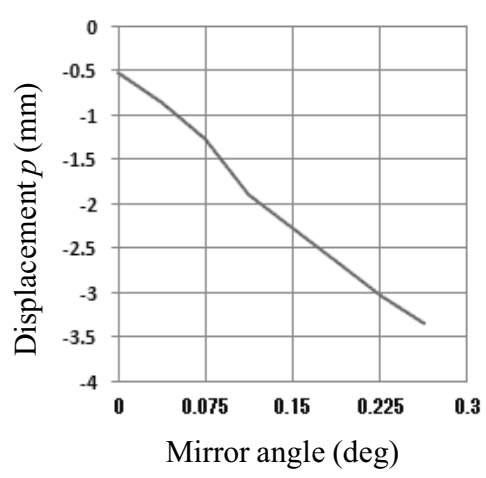

(a)

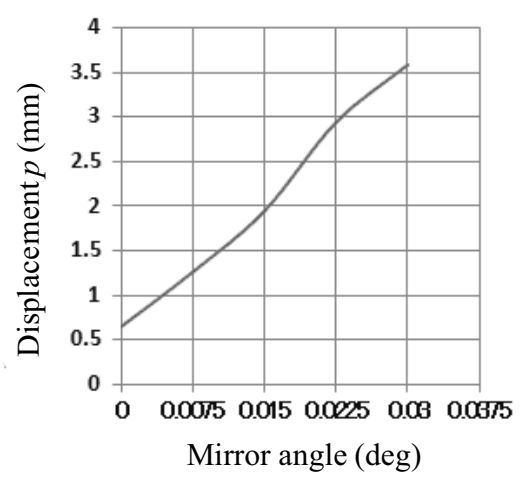

(b)

Fig. 8. Sensitivity of the mirror angle measurement: (a) without objective lens, (b) with objective lens.

\subsection{Torsion mirror fabrication process}

A torsion mirror was fabricated by the MEMS process as shown in Fig. 9. An SOI wafer $(R=1-10 \Omega \mathrm{cm})$ was oxidized and the surface $\mathrm{SiO}_{2}$ was patterned by etching [Fig. 9(a)]. The torsion mirror pattern was fabricated by dry etching the device layer [Fig. 9(b)]. Then, the $\mathrm{SiO}_{2}$ of the back surface was patterned for etching the silicon substrate [Fig. 9(c)], which was dry etched by the Bosch process or anisotropic wet etching [Fig. 9(d)]. The surface $\mathrm{SiO}_{2}$ layers on both surfaces were removed using hydrofluoric acid solution 


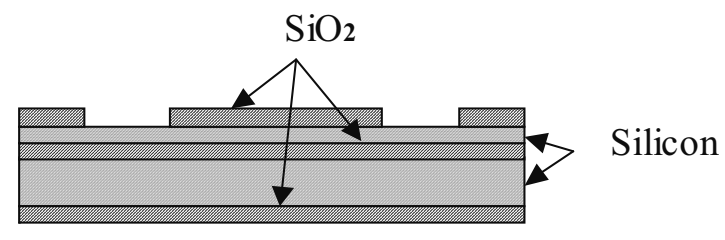

(a)

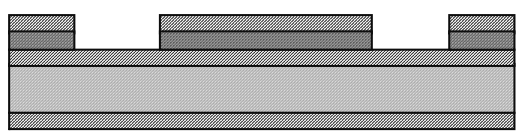

(b)

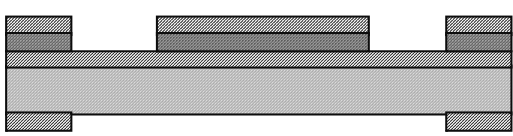

(c)

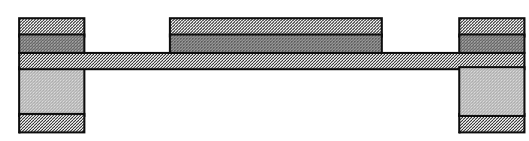

(d)

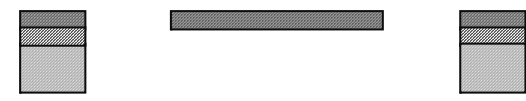

(e)

Fig. 9. Fabrication of torsion mirror.

[Fig. 9(e)]. In this fabrication, many torsion mirrors were destroyed during the silicon substrate etching [Fig. 9(d)]. Stress caused by the buried $\mathrm{SiO}_{2}$ layer bended the silicon substrate and broke it when the substrate etching was almost finished. The fact that small torsion mirrors were not destroyed in the same process show that the accumulated stress at the torsion mirror surrounding area is the main cause of destruction. To prevent such phenomena, the chosen $\mathrm{SiO}_{2}$ layers that sandwich the surface silicon layer were almost of the same thickness, and the stress caused by the upper and lower $\mathrm{SiO}_{2}$ layers was balanced. A good yield of the torsion mirror was obtained by the process. After the wafer fabrication, each torsion mirror was taken out by stealth dicing.

\subsection{Electrostatic field measurement by micromirror}

When the micromirror is moved by the nearby electrode, ${ }^{(10-12)}$ the deflection angle of the torsion mirror by electrostatic force was determined by the method of Petersen. ${ }^{(10,11)}$ When the electrostatic voltage applied to half of the micromirror is $V$, torque $T$ of the torsion mirror is given by integrating the electrostatic force over half of the micromirror, 


$$
T=\int_{0}^{b / 2} F(x) x \mathrm{~d} x \cong \frac{\varepsilon_{0} V^{2} b^{3}}{16 d^{2}}
$$

where $\varepsilon_{0}$ is dielectric constant of vacuum, $V$ is applied voltage, $b$ is mirror width, and $d$ is distance between the mirror and voltage-applying electrode. The structure and parameters of the torsion mirror are shown in Fig. 10. The deflection angle of the torsion mirror by electrostatic torque is given by

$$
\varphi=\frac{T(l / 2)}{G\left(\mathrm{~K} t^{4}\right)} \cong \frac{\varepsilon_{0} V^{2} l b^{3}(1+v)}{16 \mathrm{~K} E d^{2} t^{4}} A,
$$

where $l$ is torsion bar length, $t$ is torsion bar width and thickness, $G$ is modulus of rigidity, $v$ is Poisson's ratio, and $\mathrm{K}$ is a constant defined by the shape of the torsion bar. In the case of the torsion bar with a rectangular cross section, $\mathrm{K}$ is 0.141 (9) $^{(9)} \mathrm{\text {is }}$ Young's modulus, and $A$ is a correction factor determined using the effective electrode area. By substituting the material constant and sizes of the torsion mirror into eq. (7), the relationship between the deflection angle $\varphi$ and applied voltage at the electrostatic field is given. When $\theta$ is measured using the optical measurement system, the electrostatic voltage can be calculated from eqs. (3), (4), (6), and (7).

In the case of the charged body with an arbitrary shape and distance, the numerical simulation that includes both mechanical and electrostatic analyses will be necessary.

When an electrostatic field is applied to half of the torsion mirror, it develops not only rotation but also translation. ${ }^{(10,11,13)}$ Finite element analysis by ANSIS was carried out on the torsion mirror displacement. Figure 11 shows the results of the mode analysis, and translation modes [11(a), 11(b)] have lower resonance frequency than the rotational mode [11(c)]. In the proposed optical measurement system, however, an electrostatic force is applied to half of the micromirror and only the rotation of the torsion mirror

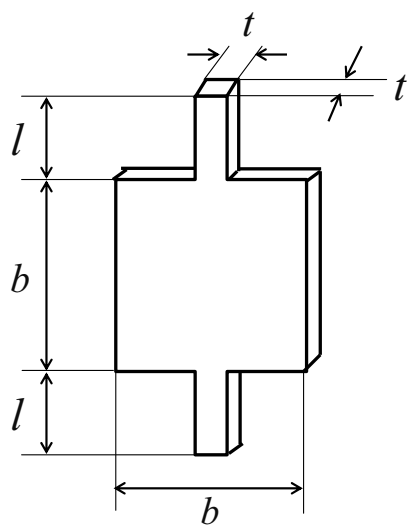

Fig. 10. Parameters of torsion mirror. 


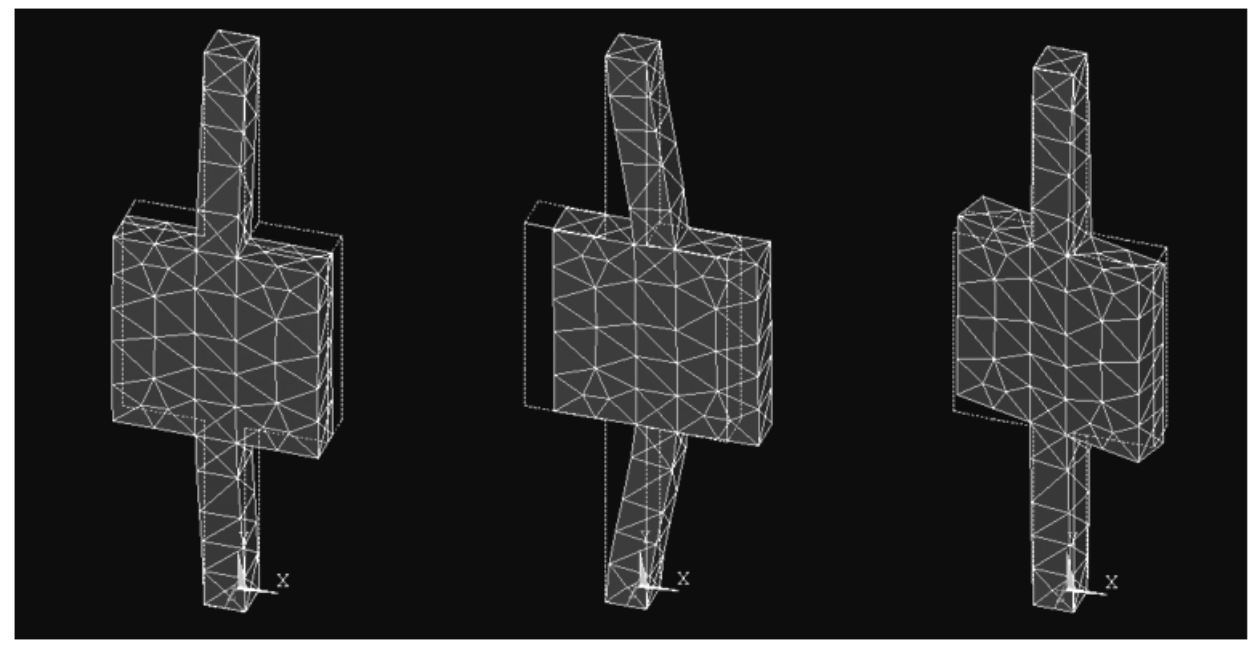

(a)

(b)

(c)

Fig. 11. Mode analysis of torsion mirror displacement. (a) $f=163.040 \mathrm{kHz}$, (b) $f=173.181 \mathrm{kHz}$, and (c) $f=238.0083 \mathrm{kHz}$.

can be detected, because the position $p$ is not changed by the translational motion of the micromirror.

Figure 12 shows a signal of the PSD output $\left(X_{1}\right)$, which was measured by scanning a laser beam on one of the torsion mirrors. The PSD can be used to measure a position of the light spot on its surface from each current flow of four electrodes. The signal was amplified using an operational amplifier and showed a good $\mathrm{S} / \mathrm{N}$ ratio.

Displacement $p$ is calculated with eq. (8) by using each electrode current output $X_{1}$, $X_{2}, Y_{1}, Y_{2}$,

$$
p=\frac{X_{2}-X_{1}+Y_{1}-Y_{2}}{X_{2}+X_{1}+Y_{1}+Y_{2}} \cdot \frac{L}{2},
$$

where $L$ is the lateral length of the PSD surface.

A high-voltage field was applied on the half surface of the torsion mirror, and reflection positions of the laser beam were measured with the PSD using the optical measurement system shown in Fig. 5. Figure 13 shows the setup for torsion mirror measurement. To prevent electric shock during measurement, a $10 \mathrm{M} \Omega$ resistor was inserted between the electrode and the high-voltage power supply. The relationship between applied voltage $V$ and deflected laser beam position $p$ on the PSD is shown in Fig. 14. When the deflection of the torsion mirror is small, $\tan \theta \approx \theta, p$ is proportional to $V^{2}$, which coincided with eq. (7). The parameters of the torsion mirror are shown in Table 1. In the measurement, the area of the high-voltage electrode was smaller than the half 


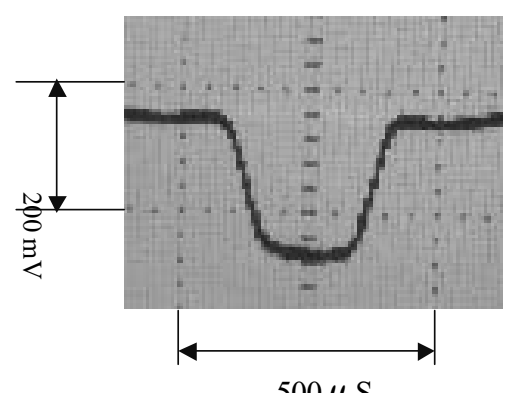

$500 \mu \mathrm{S}$

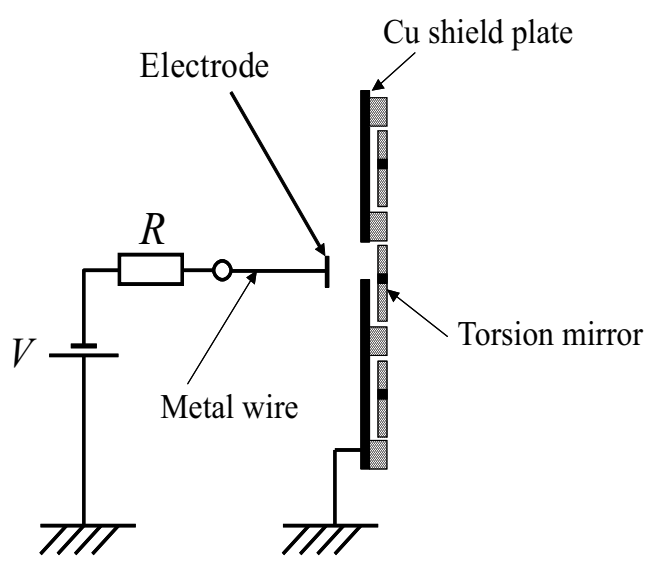

Fig. 12. (left). Output $\left(X_{1}\right)$ of PSD.

Fig. 13. (right). Setup for torsion mirror measurement.

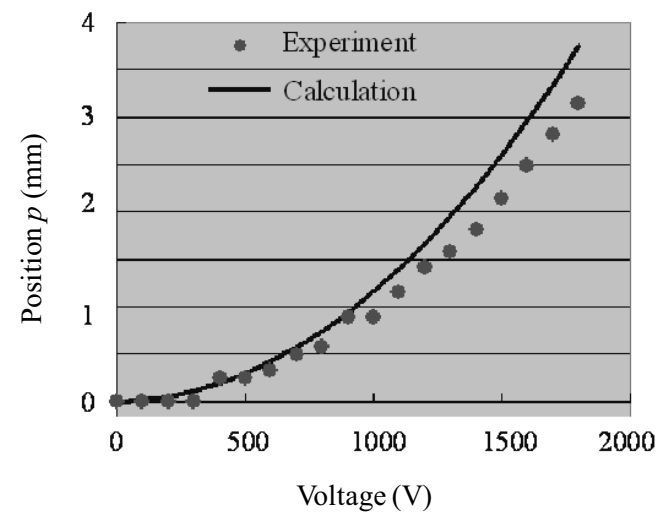

Fig. 14. Applied voltage and PSD output.

Table 1

Parameters of measurement system.

\begin{tabular}{ll}
\hline Micromirror width & $b=5 \mathrm{~mm}$ \\
Micromirror thickness & $t=20 \mu \mathrm{m}$ \\
Torsion bar length & $l=2 \mathrm{~mm}$ \\
Torsion bar width & $t=20 \mu \mathrm{m}$ \\
Torsion bar thickness & $t=20 \mu \mathrm{m}$ \\
Young's modulus & $E=169.7 \mathrm{GPa}$ \\
Poisson's ratio & $v=0.066$ \\
Rectangular shape factor & $K=0.141$ \\
Distance of electrode & $d=4 \mathrm{~mm}$ \\
Area correction factor & $A=0.17$ \\
\hline
\end{tabular}


surface of the micromirror; the correction factor $A$ of 0.17 was used. From the above results, the torsion mirror array and the proposed optical measurement can be used for measuring the electrostatic field distribution.

\section{Application of Electrostatic Distribution Measurement System to Human Body}

The spatial resolution of each torsion mirror was measured using a thin metal wire, which moved along the torsion mirror surface. A 0.5 -mm-thick copper plate with holes, where each hole was open at half of each torsion mirror, was placed at the silicon wafer with fabricated torsion mirrors. A thin metal wire was connected to a high-voltage power supply and scanned at $2.5 \mathrm{~mm}$ distance along the copper plate; then, outputs of the PSD corresponding to one torsion mirror were measured as shown in Fig. 15. To prevent electric shock during measurement, a $10 \mathrm{M} \Omega$ resistor was inserted between the thin wire and the high-voltage power supply. Figure 16 shows the output change when the metal thin wire was scanned along the torsion mirror. The spatial resolution is defined by the half width of $x$ and about $4.5 \mathrm{~mm}$ was obtained from the results. An experiment of frictional electricity was also carried out using a frictional electricity generator as shown in Fig. 17. The output of the generator was connected to the metal thin wire and the electrostatic field was measured using the spatial resolution measurement device shown in Fig. 15. The output showed the same distribution and almost the same value as the experiment shown in Fig. 15. From these results, the static field strength caused by the frictional electricity generator corresponds to the field by about $3,000 \mathrm{~V}$ of the power supply. The frictional electric field strength gradually decreased with time. These results show that it is possible to measure the electrostatic distribution of a charged body or thing using the proposed system. As the next stage, more torsion mirrors will be arrayed and the correlation between the conventional surface voltage meter and the proposed system will be checked.

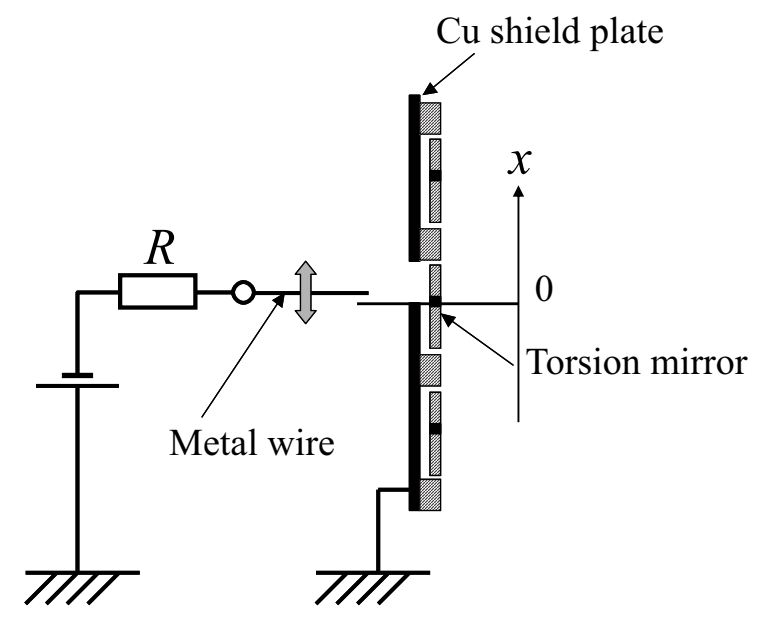

Fig. 15. Spatial resolution measurement device. 


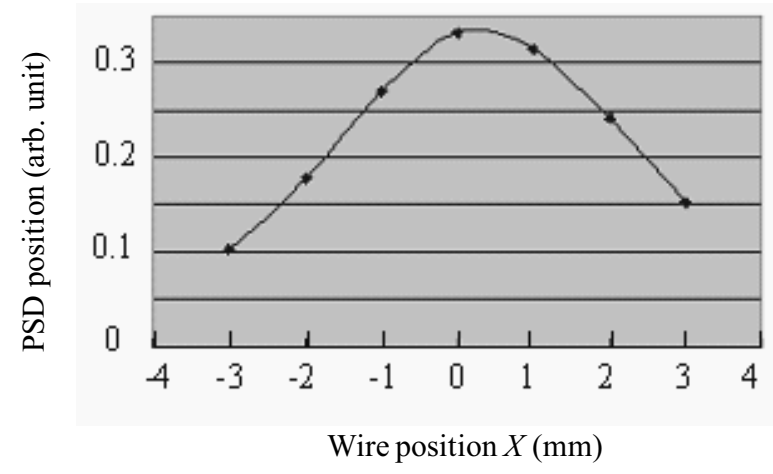

Fig. 16 Spatial resolution of electrostatic field measurement.

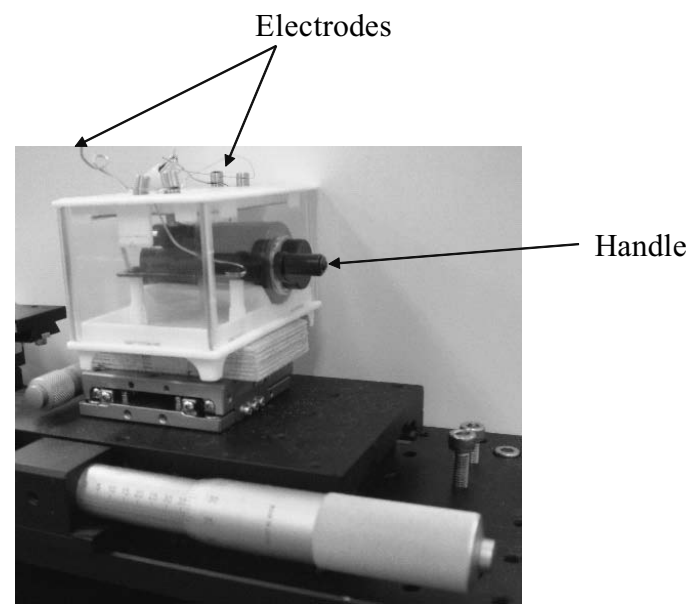

Fig. 17. Friction electricity generator.

\section{Conclusions}

The combination of the torsion mirror array made of an SOI wafer and an optical measurement system, which can be used to measure the deflection of each torsion mirror using a two-dimensional optical scanner and PSD, has enabled electrostatic distribution measurement. It was applied to a charged human body model and revealed the electrostatic field caused by using a frictional electricity generator like the human body.

\section{Acknowledgement}

This work was partially supported by the Japan Science and Technology Agency. 


\section{References}

1 J. G. Webster ed.: The Measurement, Instrumentation and Sensors Handbook (CRC Press, Boca Raton, 1999) 47-1.

2 T. R. Foord: J. Phys. E: Sci. Instrum. 2 (1969) 411.

3 C. H. Hsu and R. S. Muller: Digest of Transducers '91, (1991) 659.

4 M. N. Horenstein and P. R. Stone: J. Electrostat. 51 (2001) 515.

5 P. S. Riehl, K. L. Scotte, R. S. Muller, R. T. Howe and J. A. Yasaitis: J. Microelectromech. Syst. 12 (2003) 577.

6 C. Peng, X. Chen, Q. Bai, L. Luo and S. Xia: Proc. Int. Conf. on MEMS, Istanbul (2006) 698.

7 G. Meyer and N. M. Amer: Appl. Phys. Lett. 53 (1988) 1045.

8 G. Meyer and N. M. Amer: Appl. Phys. Lett. 56 (1990) 2100.

9 R. J. Roark and W. C. Young: Formulas for Stress and Strain (McGraw-Hill, Columbus, 1975).

10 K. E. Petersen: IBM J. Res. Dev. 124 (1980) 631.

11 K. E. Petersen: Proc. IEEE 70 (1982) 61.

12 W. Lang, H. Pavlicek, Th. Marx, H. Scheithauer and B. Schmidt: Sens. Actuators, A 74 (1999) 216.

13 J. P. Zhao and H. L. Chen: Microsyst. Technol. 11 (2005) 1301. 